\title{
Is the response to high dose oral vitamin D replacement predictable
}

\author{
D Chandrajay, M Milic, A Luvai, G Al-Khaili, A Abbas, S Orme \\ Department of Metabolic Medicine and Endocrinology, Leeds Teaching Hospitals NHS Trust, Leeds, UK
}

\section{Introduction}

Vitamin D (vitD) sufficiency is important for bone health. There are several different protocols for vitD loading recommended for treatment of vitD deficiency.

\section{Aim}

In our institution, the current protocol is to prescribe an observed oral Colecalciferol $300000 \mathrm{IU}$ for treatment of vitD deficiency (total vitD $<30 \mathrm{nmol} / \mathrm{L}$ ). VitD concentrations are then monitored at 6 weeks and 3 months post loading dose. We audited the follow up of all patients, over 12 months, who received 300000 units oral Colecalciferol, with the established local protocol.

\section{Method}

Demographic data of all the patients who received observed Colecalciferol in the unit was collected from our database. Their $\mathrm{BMI}$, age, medical history and biochemistry results were obtained from the hospital notes and results server. Data was analysed using Microsoft@ excel and Graphpad Prism® software.

\section{Results}

- A total of 256 patients (females 179: males 77) had high dose (observed) Colecalciferol. The age range was from 18-91 years old. The median age was 54.62 years.

- The commonest indications for vitD replacement were osteoporosis and osteopenia (32.5\%), biochemical vitamin D deficiency ( $21.2 \%)$, secondary hyperparathyroidism (12.9\%), fatigue $(16.9 \%)$, myalgia and muscle weakness $(9.1 \%)$. Other indications included short bowel syndrome, pregnancy, rickets, and stress fracture.

- Basal total vitD concentration ranged between $<20 \mathrm{nmol} / \mathrm{L}$ to $43.5 \mathrm{nmol} / \mathrm{L}$.

- VitD was repeated at 6 weeks, 3 months, 6 months and 12 months respectively on $63 \%, 48 \%, 49 \%$ and $58 \%$ patients.

- After 6 weeks 140/161 (87\%) had their vitD concentration $>50 \mathrm{nmol} / \mathrm{L}, 13(8.1 \%)$ had vitD concentrations between $30-50$ $\mathrm{nmol} / \mathrm{L}$ and only $8(4.9 \%)$ had vitD $<30 \mathrm{nmol} / \mathrm{L}$.

- The concentrations dropped back to $<50 \mathrm{nmol} / \mathrm{L}$ in $74 / 154$ $(48 \%)$ of the patients within 12 months.

- $48(18.75 \%)$ patients required oral reloading within 12 months.

- 7 were switched to IM vitamin D preparations.

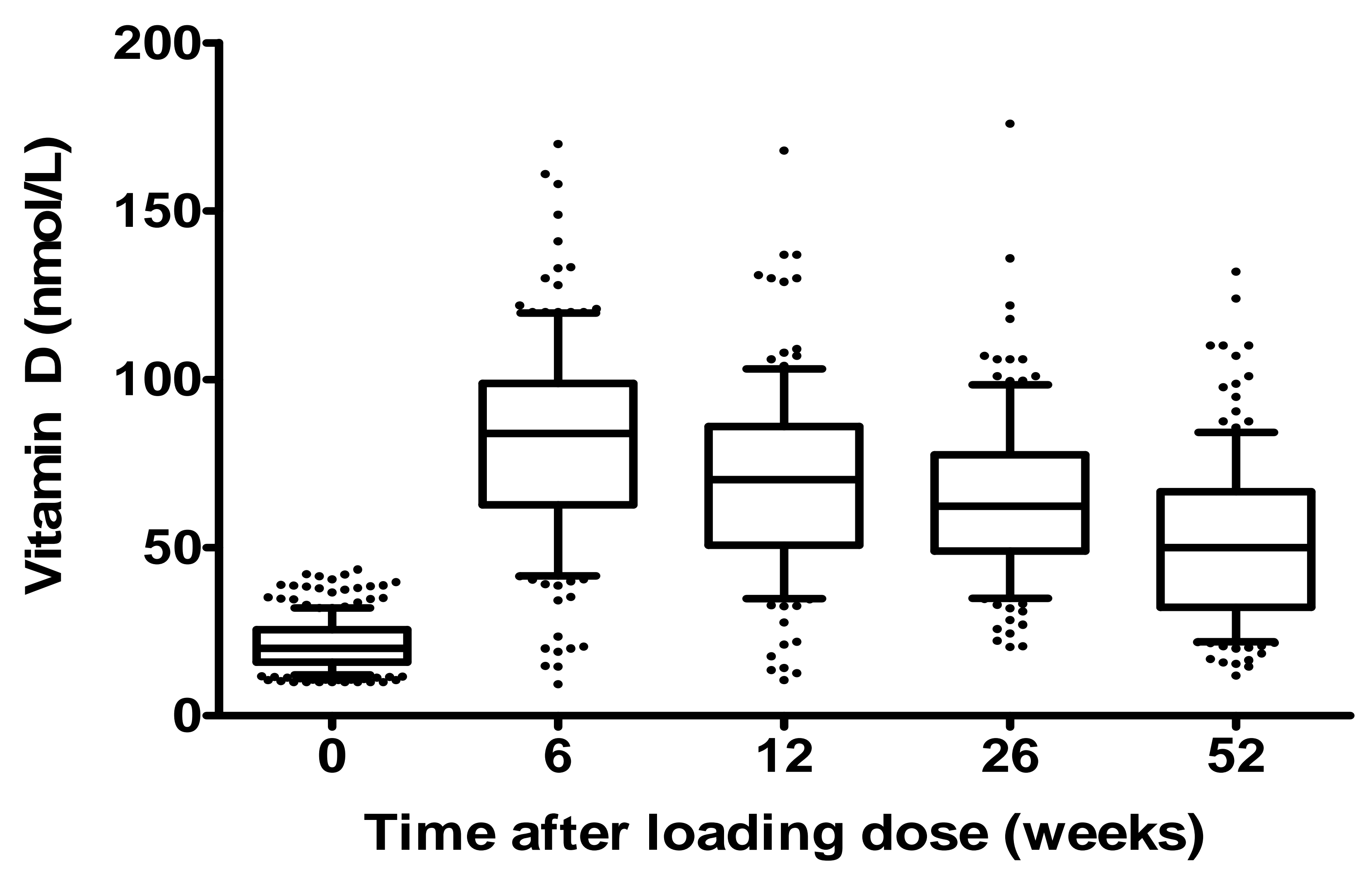

Figure 1. Response to $3000001 \mathrm{U}$ oral Colecalceferol with time

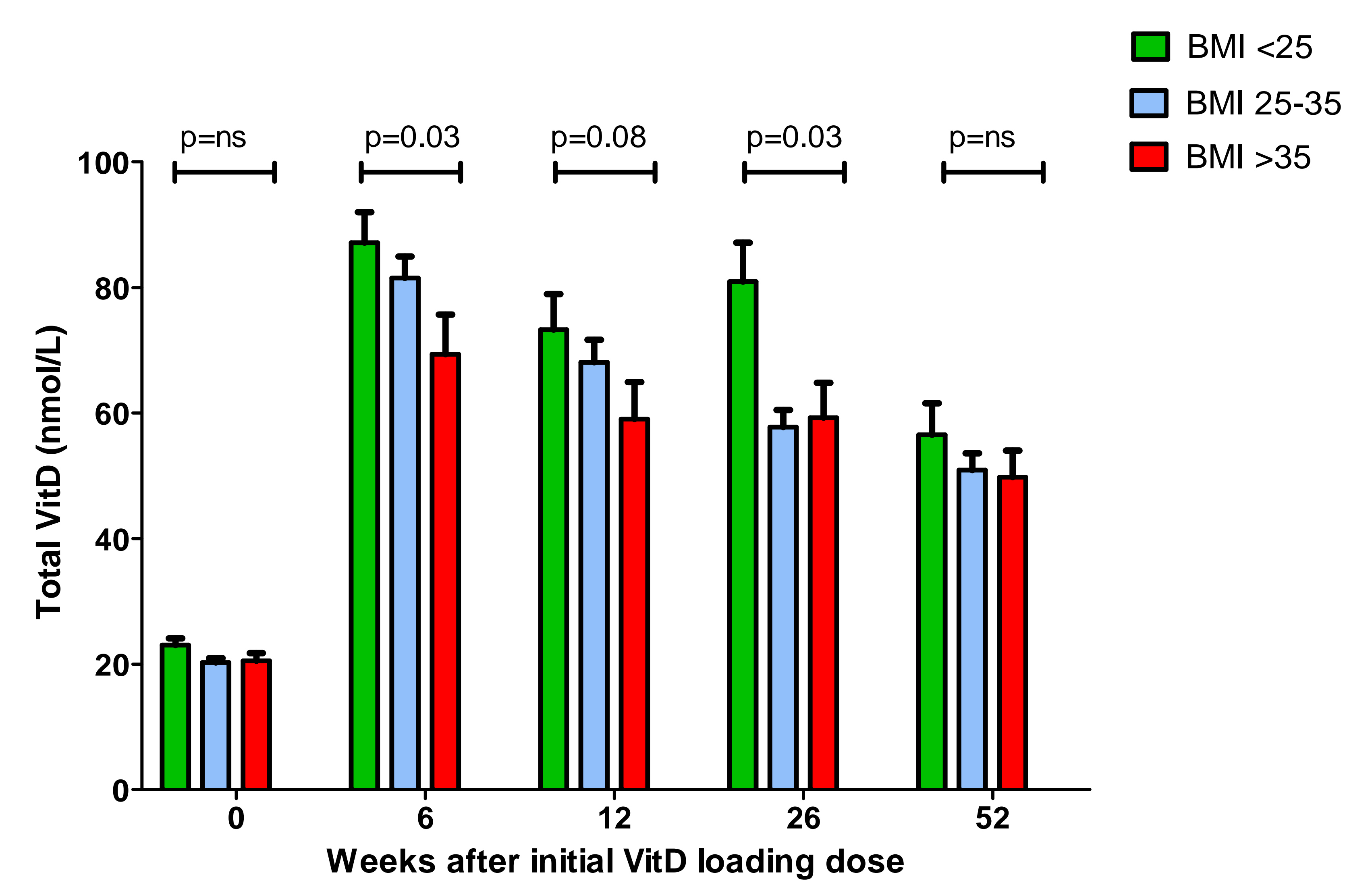

Figure 2. relationship between BMI and response to $300000 \mathrm{IU}$ oral Colecalciferol
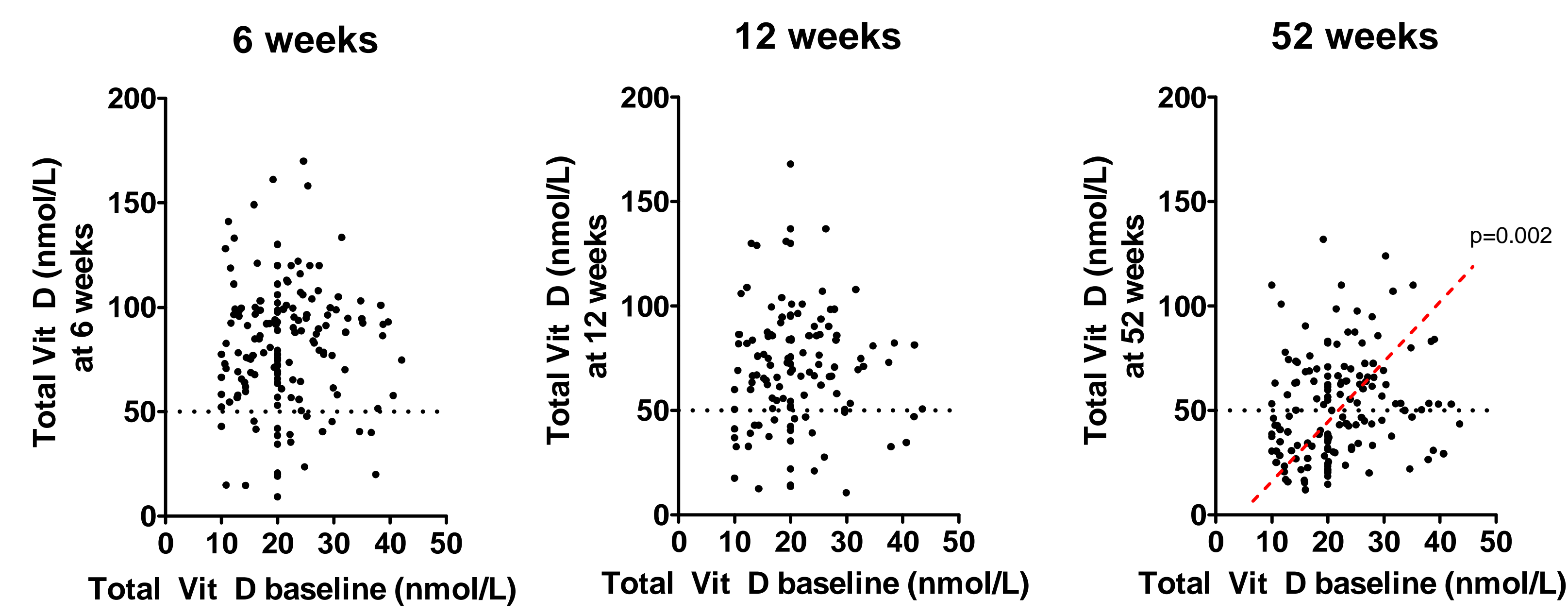

Figure 3. Relationship between baseline vitD and post dose vitD at different intervals.

No significant relationship was noted between baseline vitD concentration and response to loading dose Colecalciferol at 6 or 12 weeks. (Figure 3)

However patients with lowest baseline vitD concentration were significantly more likely to be deficient once again by 52 weeks

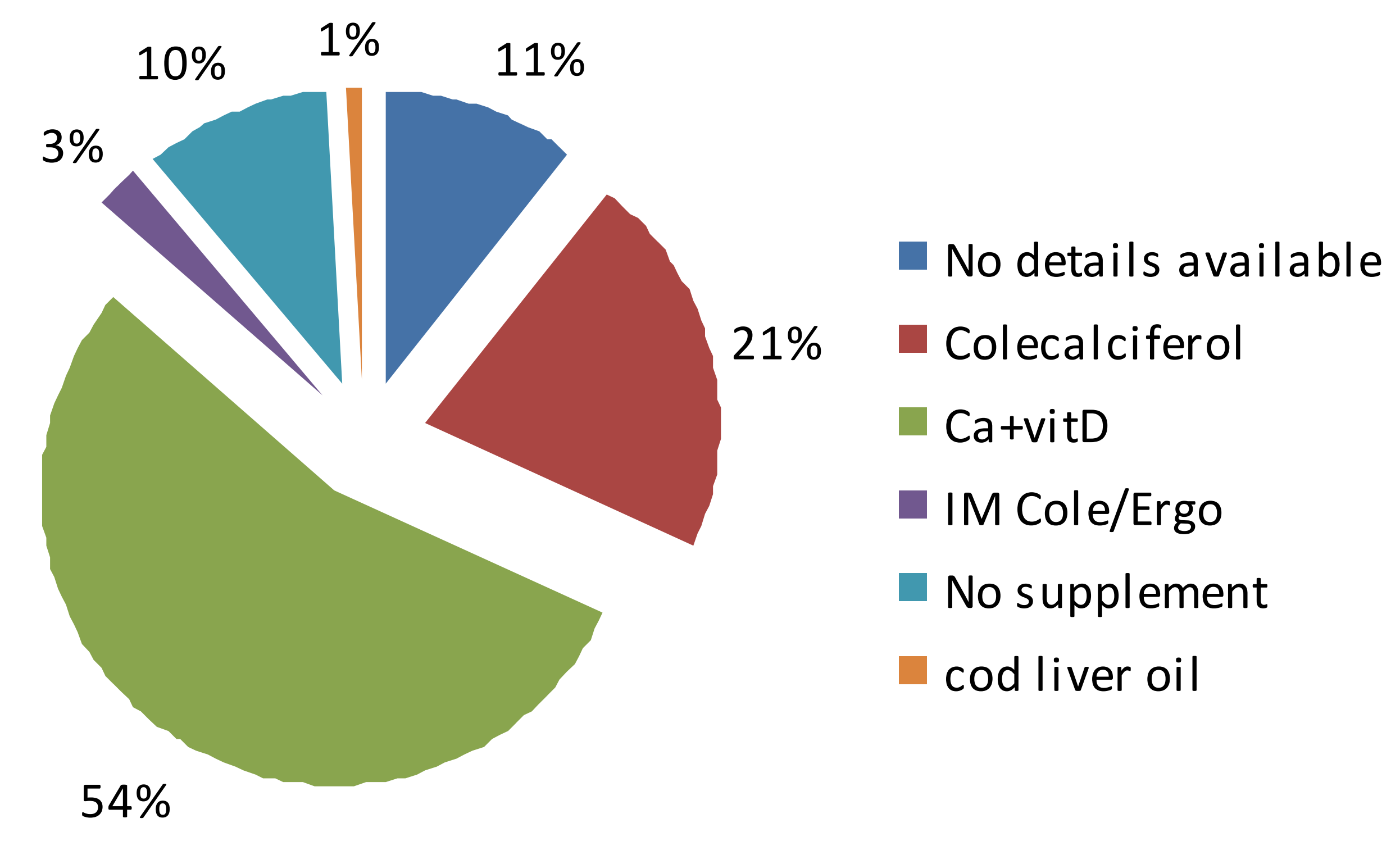

Figure 4. Follow up treatment

\section{Conclusions}

1. Most patients had a good vitD response to loading dose of 300000 IU oral Colecalciferol.

2. VitD concentrations dropped again within 12 months in nearly half of all patients, and almost one fifth of patients required reloading. Therefore regular follow-up is required.

3. The rise in vitD concentration after loading dose was significantly lower in obese patients.

4. No significant side effects including vitD toxicity or hypercalcaemia were observed in any of the patients receiving high dose Colecalciferol in our unit.

5. $57 \%$ of the patients who were switched to IM vitD replacement had coexisting malabsorption. 